\title{
Silencing of the human SET gene in vitro with lentivirus-mediated RNA interference
}

\author{
YANFENG LIU, PENGCHENG HE, MEI ZHANG，LILI SHI，HUACHAO ZHU，YUAN WANG and JING ZHAO \\ Department of Hematology, The First Affiliated Hospital, School of Medicine, \\ Xi'an Jiaotong University, Xi'an 710061, P.R. China
}

Received August 25, 2012; Accepted December 19, 2012

DOI: $10.3892 / \mathrm{mmr} .2013 .1275$

\begin{abstract}
In our previous study, SET was identified as one of the differentially expressed proteins that was associated with tetra-arsenic tetra-sulfide $\left(\mathrm{As}_{4} \mathrm{~S}_{4}\right)$-induced NB4-R1 [retinoic acid-resistant acute promyelocytic leukemia (APL) cell line] apoptosis. However, the mechanism through which SET regulates pathways during this process remains unclear. The aim of this study was to construct lentivirus-mediated short hairpin RNA (shRNA) against SET and investigate the effect of SET on $\mathrm{As}_{4} \mathrm{~S}_{4}$-induced retinoic acid-resistant APL cell apoptosis. In the present study, 4 different oligonucleotides targeting the human SET gene were synthesized and cloned into the eukaryotic expression plasmid pGCSIL-GFP. The recombinant vectors were introduced into NB4-R1 cells. The silencing efficiency was measured by real-time quantitative PCR (RT-qPCR) and western blotting. Our results showed that the 4 recombinant RNA interference (RNAi) vectors were constructed successfully. Fluorescence microscopy demonstrated that infection efficiency ranged from 70 to $90 \%$. Infection with the 4 different RNAi vectors significantly knocked down the expression of SET by 52.8 , 69.1, 48.9 and $90.3 \%$ at the mRNA level, and 92.5, 96.3, 91.7 and $98.4 \%$ at the protein level, respectively. We attempt to clarify the mechanism of $\mathrm{As}_{4} \mathrm{~S}_{4}$ treatment on retinoic acidresistant APL.
\end{abstract}

\section{Introduction}

Acute promyelocytic leukemia (APL), a particular subtype of acute myeloid leukemia (AML) with a distinct cytological morphology, is characterized by a chromosome reciprocal

Correspondence to: Dr Pengcheng He or Professor Mei Zhang, Department of Hematology, The First Affiliated Hospital, School of Medicine, Xi'an Jiaotong University, No. 277 Yanta West Road, Xi'an 710061, P.R. China

E-mail: hepc@163.com

E-mail: zhangmei@medmail.com.cn

Key words: SET, acute promyelocytic leukemia, RNA interference, lentivirus, NB4-R1 translocation $\mathrm{t}(15 ; 17)$, which results in the fusion between the promyelocytic leukemia (PML) gene and the retinoic acid receptor $(\mathrm{RAR} \alpha)$ gene $(1,2)$. During the 1980 s, the introduction of all-trans retinoic acid (ATRA) created a precedent for tumor molecular target therapy, enabling the complete remission of $>95 \%$ APL patients (3). However, due to the wide application of ATRA, drug-resistant cases are increasingly identified (4). As a result, producing a more effective treatment for ATRA-resistant APL patients has become a challenge in the clinic. Since the early 1990s, arsenic compounds have regained attention due to the discovery of their clinical effects and unique mode of action on refractory or relapsed patients, as well as patients newly diagnosed with APL $(1,5)$. Tetra-arsenic tetra-sulfide $\left(\mathrm{As}_{4} \mathrm{~S}_{4}\right)$ is a type of traditional Chinese medicine. In comparison with arsenic trioxide $\left(\mathrm{As}_{2} \mathrm{O}_{3}\right), \mathrm{As}_{4} \mathrm{~S}_{4}$ has advantages including high remission rate, reduced side effects, ease of use (may be taken orally) and cost-effectiveness. At present, $\mathrm{As}_{4} \mathrm{~S}_{4}$ has become one of the most important therapies for APL (especially for recrudescent and drug-resistant APL) (6).

In our previous study, we applied proteomics to screen $>20$ differently expressed proteins before and after treatment with $\mathrm{As}_{4} \mathrm{~S}_{4}$ in the ATRA-resistant APL cell line-NB4-R1. Among these proteins, SET expression was observed to be markedly decreased following treatment with $\mathrm{As}_{4} \mathrm{~S}_{4}$ for $48 \mathrm{~h}$, and the number of apoptotic NB4-R1 cells increased significantly (7). However, the function, mechanism and regulation pathways of SET in $\mathrm{As}_{4} \mathrm{~S}_{4}$-induced apoptosis requires further investigation.

RNA interference (RNAi) is a posttranscriptional gene silencing mechanism which is mediated by double-stranded RNA (dsRNA) molecules. RNAi is a new, powerful and widely used tool for the analysis of gene function in mammals and plants $(8,9)$. Lentiviral vector-based RNAi is able to combine efficient infection and integration of lentiviral DNA with suppression of specific homologous gene expression of host RNA. Therefore, lentiviral vector-based RNAi may provide a long-lasting knockdown influence (10). In the present study, we used powerful lentiviral-mediated RNAi eukaryotic vectors with specific short hairpin RNA (shRNA) to block the expression of SET, in order to lay the foundation for further investigation of its mechanism in $\mathrm{As}_{4} \mathrm{~S}_{4}$-induced APL cell apoptosis, and provide new insight into the pharmacological actions of traditional medicines. 


\section{Materials and methods}

Cell culture. The NB4-R1 cell line was donated by Shanghai Jiao Tong University School of Medicine. The cells were cultured in RPMI-1640 (Gibco-BRL, Carlsbad, CA, USA) supplemented with $10 \%$ heat-inactivated fetal bovine serum at $37^{\circ} \mathrm{C}$ in a humidified incubator containing $5 \% \mathrm{CO}_{2}$. Cell viability was evaluated by the trypan blue dye exclusion assay and only cell suspensions that presented $>95 \%$ viability were used. The study was approved by the ethics committee of Xi'an Jiaotong University, Xi'an, China.

Design and clone of RNAi lentiviral vectors. Four target shRNAs against human SET gene (Genbank accession NM_003011) for RNAi were designed using the internet application Ambion (Austin, TX, USA) (Table I). The sequence 5'-TTCTCCGAACGTGTCACGT-3' which had no significant homology to any known human genes was used as a negative control. Oligonucleotides were synthesized according to the sequences, heated at $95^{\circ} \mathrm{C}$ for $5 \mathrm{~min}$ and then annealed at $37^{\circ} \mathrm{C}$ for $1 \mathrm{~h}$. Annealed sequences were ligated into the AgeI and EcoRI sites of pGCSIL-GFP (containing human U6 promoter) to generate pGCSIL-GFP-SET (1-4) vectors (Shanghai Genechem Co., Shanghai, China), which were transformed into E. coli. Positive recombinant clones were selected by PCR and DNA sequencing. The new recombinant lentiviral vectors co-expressed the shRNA transcript and green fluorescent protein (GFP).

Lentiviral vector infection. Cells were divided into 3 groups: CON (normal NB4-R1 cells), NC (infection with negative control RNAi vector), and KD (infection with pGCSIL-GFPSET). According to different shRNA sequences, the KD group was divided into 4 subgroups: KD1, KD2, KD3 and KD4.

The NB4-R1 cells were plated into 6-well plates at a density of $1 \times 10^{5}$ cells $/ \mathrm{ml}$. The following day, cells were infected with a mixture of $100 \mu 12 X$ polybrene and specific negative control lentiviral vectors, at the multiplicity of infection (MOI) 20 , according to the preliminary results. The final volume of culture medium was $1 \mathrm{ml} /$ well. After centrifugation for $18 \mathrm{~h}$, the supernatant was discarded and the precipitate was resuspended in the culture medium with $10 \%$ FBS. The cells were incubated, observed under fluorescence microscopy and harvested on the 4th and 7th day after infection. The knockdown efficiency of SET was analyzed by real-time quantitative PCR (RT-qPCR) and western blotting.

$R T-q P C R$. After infection with lentiviruses for 4 days, the total RNA from each group of cells was extracted in TRIzol (Invitrogen, Carlsbad, CA, USA) and quantified by an ultraviolet spectrophotometer at a wavelength of $260 \mathrm{~nm}$. The reverse transcription (RT) reaction was performed with a RevertAid First Strand cDNA Synthesis kit (Fermentas, Burlington, ON, Canada) according to manufacturer's instructions. The PCR conditions were as follows: initial denaturation at $94^{\circ} \mathrm{C}$ for $5 \mathrm{~min}$, the reaction was repeated for 45 cycles, each cycle consisted of denaturing at $94^{\circ} \mathrm{C}$ for $30 \mathrm{sec}$, annealing at $61^{\circ} \mathrm{C}$ for $45 \mathrm{sec}$ and synthesis at $72^{\circ} \mathrm{C}$ for $45 \mathrm{sec}$. The primer sequences used to amplify SET and GAPDH are listed in Table II. The cutoff point $(\mathrm{Ct})$ of each sample was plotted on
Table I. shRNA sequence.

\begin{tabular}{ll}
\hline Marker & \multicolumn{1}{c}{ Target sequence } \\
\hline KD-1 & GAGTTCACAGAAGCGGTGGAA \\
KD-2 & GCTGCCGTCCATCACAACTGA \\
KD-3 & CCGTGGGTACAGAAACCAATT \\
KD-4 & CGTGGTGAACTCTGCCTTATA
\end{tabular}

shRNA, small hairpin RNA.

the standard curve and the mRNA copy numbers were calcuated. The GAPDH gene was used as an endogenous control. The relative SET mRNA levels were expressed as a ratio of SET to GAPDH.

Western blotting. After infection with lentiviruses for 7 days, the cells were lysed in RIPA buffer in the presence of proteinase inhibitor cocktail (Sigma, Santa Clara, CA, USA). The protein concentration was determined by BCA assay kit (Thermo, Waltham, MA, USA). Protein $(30 \mu \mathrm{g})$ was separated by $12 \%$ SDS-PAGE and transferred to a nitrocellulose membrane. The membranes were blocked in 5\% skimmed milk for $2 \mathrm{~h}$, incubated with primary antibodies against SET (goat monoclonal, 1:1,000; Abcam, Cambridge, MA, USA) and GAPDH (mouse monoclonal, 1:10,000; Santa Cruz Biotechnology, Inc., Santa Cruz, CA, USA) at room temperature for $2 \mathrm{~h}$ and $4^{\circ} \mathrm{C}$ overnight. The membranes were washed with TBS containing $0.1 \%$ Tween-20 (TBST) followed by an incubation of $1 \mathrm{~h}$ in goat anti-mouse and goat anti-goat secondary antibody conjugated with HRP. After final washing with TBST, the membranes were developed using chemiluminescence and exposed to $\mathrm{X}$-ray films. The immunoblots were quantified with software Quantity One version 4.6.2. The expression of SET in each sample was internally normalized to GAPDH and levels were expressed relative to expression in control groups.

Statistical analysis. All the experiments were carried out in triplicate and quantitative data in this study were expressed as mean \pm SD. Statistical differences between groups were compared using a one-way analysis of variance (ANOVA). $\mathrm{P}<0.05$ was considered to indicate a statistically significant result.

\section{Results}

Construction and packaging of lentiviral vectors. Four target shRNAs against human SET gene for RNAi were constructed and ligated with lentiviral frame plasmids. Positive recombinant clones were selected and identified with PCR. The sizes of PCR amplified fragments of recombinant clones and unconnected shRNA empty vectors were 343 and $306 \mathrm{bp}$, respectively (Fig. 1A). Sequence analysis confirmed that the inserted SET shRNA sequences were correct (Fig. 1B). The recombinant lentiviral vectors were applied to infect NB4-R1 cells and virus titers were determined with the hole by hole dilution method as $1 \times 10^{9}(\mathrm{KD} 1), 3 \times 10^{8}(\mathrm{KD} 2), 4 \times 10^{8}(\mathrm{KD} 3)$ and $6 \times 10^{8}(\mathrm{KD} 4) \mathrm{TU} / \mathrm{ml}$, respectively. 
Table II. Forward (F) and reverse (R) primer sequences for real-time quantitative PCR.

\begin{tabular}{llc} 
Target gene & \multicolumn{1}{c}{ Primer sequence } & Amplification length \\
\hline SET-F & 5'-AAATATAACAAACTCCGCCAACC-3' & 138 bp \\
SET-R & 5'-CAGTGCCTCTTCATCTTCCTC-3' & 121 bp \\
GAPDH-F & 5'-CACCCTGTTGCTGTAGCCAAA-3' \\
GAPDH-R & 5'-CACCCTGTTGCTGTAGCCAAA-3'
\end{tabular}

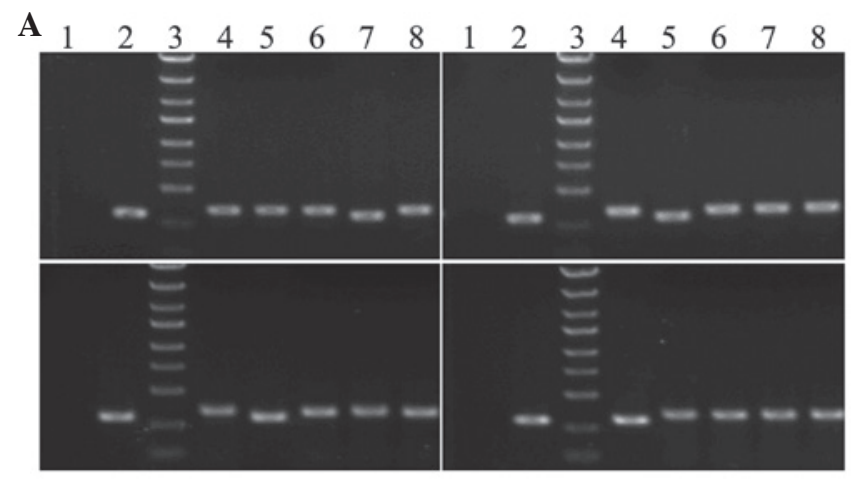

B
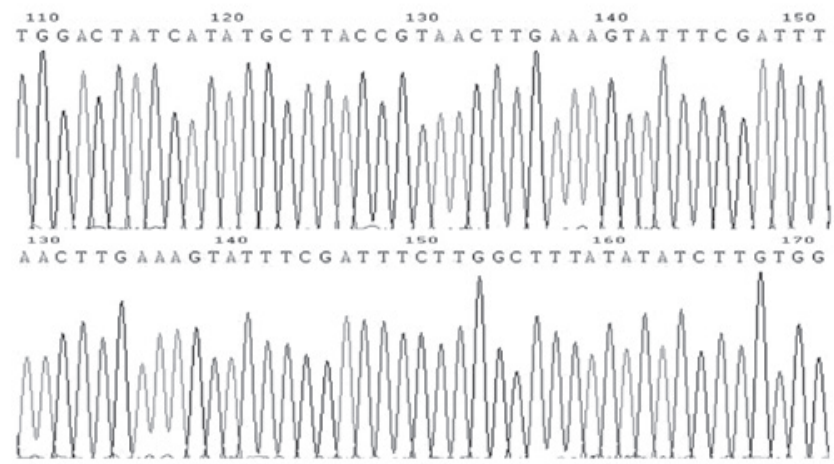

TTCTGTCACTTCAACTCTGGTTTTTGAATTCGGATCCATTAGG

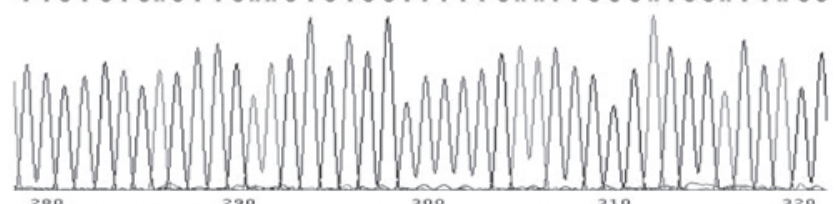

CGTATTACC GCCATGCATTAGTTATTAATA GTA ATCA A TTACG

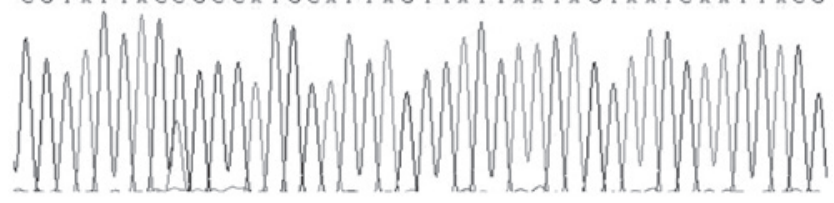

Figure 1. (A) Identification of recombinant lentiviral vectors by PCR. Lane 1: normal control; Lane 2: recombinant negative lentiviral vector (343 bp); Lane 3: marker ladder (from top to bottom 5000, 3000, 2000, 1500, 1000, 750, 500, 250, $100 \mathrm{bp}$ ); Lanes 4-8: recombinant positive lentiviral vector with SET shRNA (343 bp). (B) Identification of recombinant lentiviral vectors by sequence analysis.

Infection efficiency of NB4-R1 cells with lentivirus. The assessment of infection rate was accomplished with the determination of positive expression rate of GFP by fluorescent microscopy $72 \mathrm{~h}$ after infection. As shown in Fig. 2, the cell growth state was good, and the proportion of fluorescent cells ranged from $70-90 \%$ in each group, with the exception of the

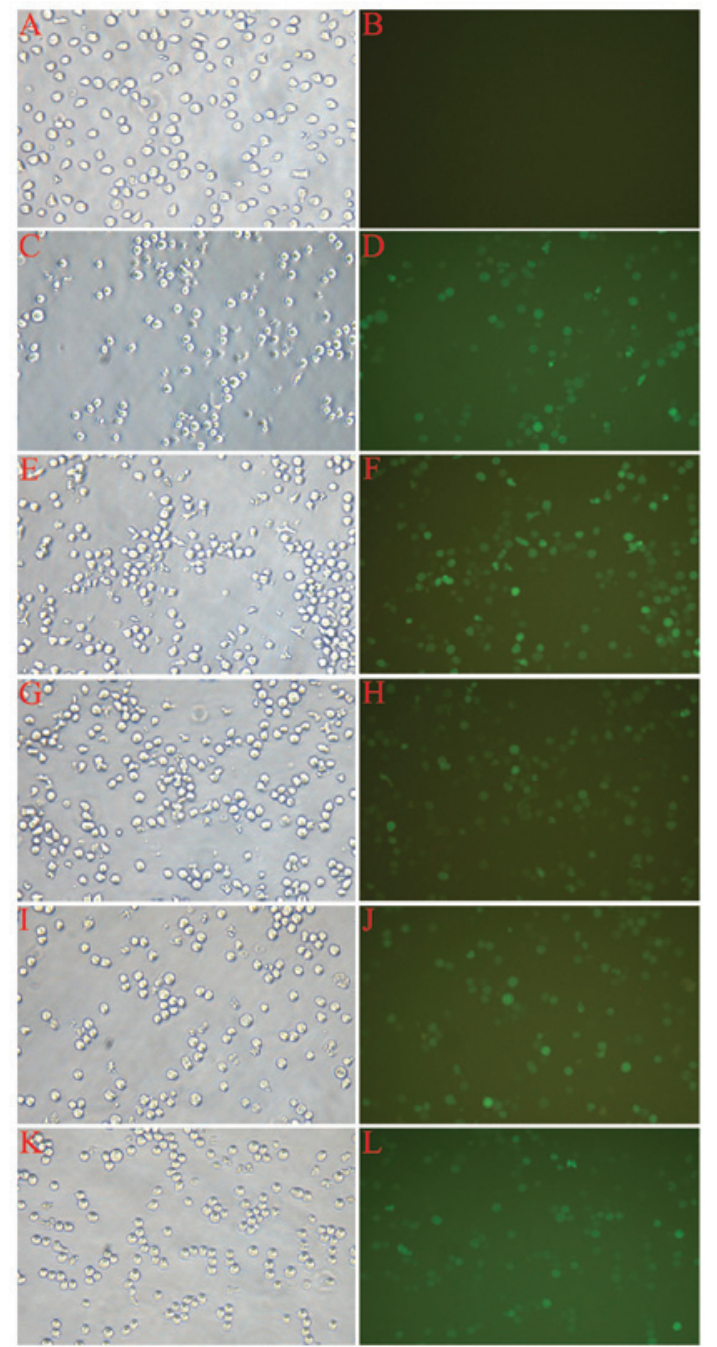

Figure 2. Fluorescence images of NB4-R1 cells following lentiviral vector infection (magnification $\mathrm{x} 100)$. (A and $\mathrm{B})$ normal control (CON, without lentiviral vector); (C and D) negative control (NC, NB4-R1 + negative lentiviral vector); (E and F) KD1 (NB4-R1 + KD1 lentiviral vector with shRNA of SET); (G and H) KD2 (NB4-R1 + KD2 lentiviral vector with shRNA of SET); (I and J) KD3 (NB4-R1 + KD3 lentiviral vector with shRNA of SET); (K and L) KD4 (NB4-R1 + KD4 lentiviral vector with shRNA of SET). shRNA, small hairpin RNA.

normal control (CON). The cells were then harvested for the following experiments.

Silencing efficacy of SET at mRNA level. The silencing efficacy of SET shRNA lentivirus at the mRNA level in NB4-R1 cells was determined by RT-qPCR. As shown in Fig. 3A, infection 
A
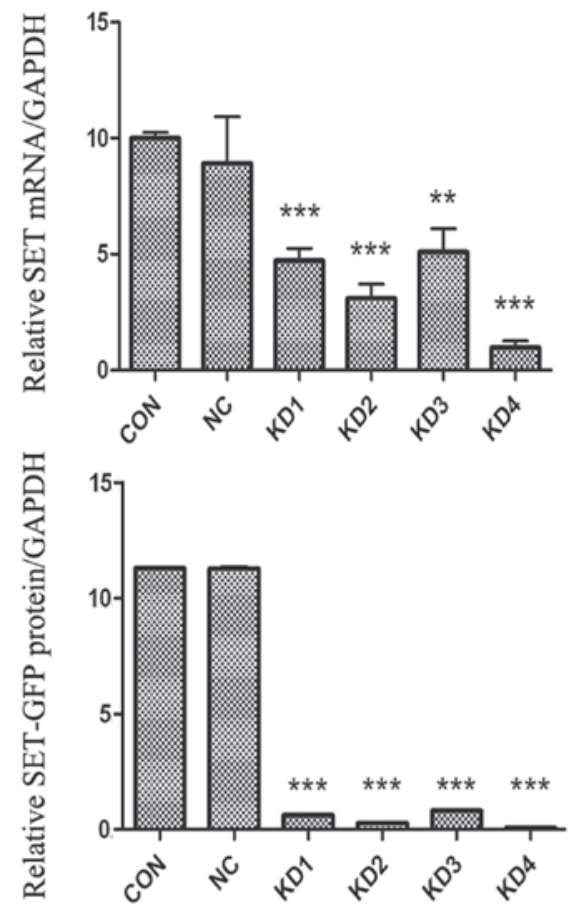

B

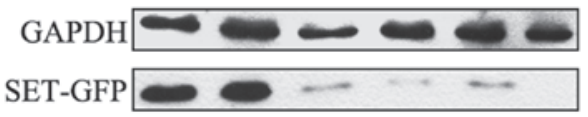

Figure 3. Expression of SET mRNA and protein in NB4-R1 cells after infection with lentivirus. (A) The relative level of SET mRNA in NB4-R1 cells after infection with lentivirus. CON, normal control without lentiviral vector; NC, negative control NB4-R1 + negative lentiviral vector; KD1, NB4-R1 + KD1 lentiviral vector with shRNA of SET; KD2, NB4-R1 + KD2 lentiviral vector with shRNA of SET; KD3, NB4-R1 + KD3 lentiviral vector with shRNA of SET; KD4, NB4-R1 + KD4 lentiviral vector with shRNA of SET. ${ }^{* *} \mathrm{P}<0.01 ;{ }^{* * * *} \mathrm{P}<0.001$. (B) The immunoblot of SET in NB4-R1 cells following infection with lentivirus.

of NB4-R1 cells with SET shRNA resulted in a significant decrease in mRNA expression of SET. At the 4th day after infection, the amount of SET mRNA in KD1-KD4 subgroups was notably reduced by $52.8,69.1,48.9$ and $90.3 \%$, respectively, compared with that of $\mathrm{NC}$ group. However, there was no difference between the $\mathrm{NC}$ and $\mathrm{CON}$ groups.

Silencing efficacy of SET at protein level. Western blot analysis of cell extracts was carried out to determine whether decreased mRNA expression was correlated with decreased translation of the gene product. As shown in Fig. 3B, a similar trend was observed in NB4-R1 cells. The protein expression levels of SET in KD1-KD4 subgroups were decreased by 92.5 , 96.3, 91.7 and $98.4 \%$, respectively, compared to the NC group. However, there was no significant difference in the protein expression level between the NC and CON groups. RT-qPCR and western blotting demonstrated that lentiviral vectors were effective for SET silencing and KD4 was the most effective construct. Stable infection was performed with KD4 construct.

\section{Discussion}

Acute promyelocytic leukemia (APL) is one of the most common malignancies and has a high mortality rate. The comprehensive treatments have been constantly improved; for example, ATRA may enable the complete remission of $>95 \%$ of APL patients (3). However, an increasing resistance trend restricted the long-term efficacy of ATRA (11). In recent years, arsenic compounds (mainly $\mathrm{As}_{2} \mathrm{O}_{3}$ and $\mathrm{As}_{4} \mathrm{~S}_{4}$ ) have gradually regained attention due to their positive clinical effects and unique mode of action on APL (12). However, a disadvantage of $\mathrm{As}_{2} \mathrm{O}_{3}$ is that the daily intravenous infusion required to achieve the ideal therapeutic result causes dermal and gastrointestinal side effects (13). $\mathrm{As}_{4} \mathrm{~S}_{4}$, another arsenic compound, as a type of traditional Chinese medicine (also named realgar or red orpiment), has been shown to be equally effective in the treatment of APL, with less toxicity and side effects, and $\mathrm{As}_{4} \mathrm{~S}_{4}$ only requires oral administration (14-16). Based on above advantages, this orally administered agent would thus provide more benefits not only for quality of life but also for easy access to consolidation and maintenance therapy for leukemia.

In our previous study, SET was identified as one of the differentially expressed proteins that correlated with $\mathrm{As}_{4} \mathrm{~S}_{4}$-induced NB4-R1 cell apoptosis. SET (also known as TAF-1 $\beta$, I2PP2A or IGAAD), localized to the nucleus and cytoplasm, is a multifunctional protein. It is clear that SET is a potent inhibitor of protein phosphatase 2A (PP2A), a phosphatase with tumor suppressor activity (17). SET has also been described as an inhibitor of the tumor suppressor NM23-H1 (a granzyme A DNase-activated factor) (18) and a negative regulator of histone acetylation (19).

PP2A is one of the major serine-threonine protein phosphatase in all eukaryotic cells (20). PP2A is involved in almost all cellular processes, including signal transduction (21), cell cycle progression (22) transcriptional and translational regulation, DNA replication and chromosome depolymerization $(23,24)$. As a potential tumor suppressor, inhibition of PP2A activity appears to be a common event in different human neoplasia. For example, oncogenic viral proteins, such as the SV40 virus small-t antigen, inhibits PP2A activity by interacting with the subunits of PP2A (25). In addition, numerous genetic alterations of the structure of PP2A subunit have been identified in several types of human cancer. Consequently, exploring the molecular targets inhibiting PP2A activity has an important prevention significance for tumor treatment.

SET is the major cellular inhibitor of PP2A, and is involved in the regulation of a variety of cellular processes and signal transduction pathways (26). Although overexpression of SET has been reported in tumor tissue in the uterus, stomach, colon and rectum, as well as chronic myelogenous leukemia $(27,28)$, its role in the regulation of APL cells remains unclear. There is evidence that SET may be involved in NB4-R1 cell apoptosis induced by $\mathrm{As}_{4} \mathrm{~S}_{4}$ in vivo. Therefore, in this study we designed 4 shRNA sequences targeting against SET and constructed 4 recombinant lentiviral vectors. After infection with NB4-R1 cells, RT-qPCR and western blotting were used to identify silencing efficiency. Our results showed that the 4 RNAi vectors all had high efficiency of infection; among these, the knockdown efficiency of KD4 was the greatest, and was chosen to conduct the follow-up research. This study will lay the foundation for further study on the function and mechanism of SET. SET may be used in the future as a potential target of gene therapy for ATRA-resistant APL and other cancer. 


\section{Acknowledgements}

This study was supported by the Natural Science Foundation of China (nos. 30701133 and 81000218) and the Fundamental Research Funds for the Central Universities and the Shaanxi Province Science and Technology Development Fund, China (nos. 2010K01-135 and 2012KTCL03-12). The authors express their gratitude to Dr Xinyang Wang and Dr Wen Wen for their technological assistance.

\section{References}

1. Wang ZY and Chen Z: Acute promyelocytic leukemia: from highly fatal to highly curable. Blood 111: 2505-2515, 2008.

2. Kogan SC: Curing APL: differentiation or destruction? Cancer Cell 15: 7-8, 2009 .

3. Hoffman E and Mielicki WP: All-trans retinoic acid (ATRA) in prevention and cancer therapy. Postepy Hig Med Dosw (Online) 64: 284-290, 2010 (In Polish).

4. Wang T, Ma X, Krausz KW, Idle JR and Gonzalez FJ: Role of pregnane $\mathrm{X}$ receptor in control of all-trans retinoic acid (ATRA) metabolism and its potential contribution to ATRA resistance. J Pharmacol Exp Ther 324: 674-684, 2008.

5. Yin T, Wu YL, Sun HP, et al: Combined effects of $\mathrm{As}_{4} \mathrm{~S}_{4}$ and imatinib on chronic myeloid leukemia cells and BCR-ABL oncoprotein. Blood 104: 4219-4225, 2004.

6. Wu J, Shao Y, Liu J, Chen G and Ho PC: The medicinal use of realgar $\left(\mathrm{As}_{4} \mathrm{~S}_{4}\right)$ and its recent development as an anticancer agent. J Ethnopharmacol 135: 595-602, 2011.

7. Qi J, He PC, Chen W, Wang H, Wang X and Zhang M: Comparative proteome study of apoptosis induced by $\mathrm{As}_{4} \mathrm{~S}_{4}$ in retinoid acid resistant human acute promyelocytic leukemia NB4-R1 cells. Leuk Res 34: 1506-1516, 2010.

8. Pecot CV, Calin GA, Coleman RL, Lopez-Berestein G and Sood AK: RNA interference in the clinic: challenges and future directions. Nat Rev Cancer 11: 59-67, 2011.

9. Hannon GJ: RNA interference. Nature 418: 244-251, 2002.

10. SunBS,DongQZand YeQH,etal:Lentiviral-mediatedmiRNA against osteopontin suppresses tumor growth and metastasis of human hepatocellular carcinoma. Hepatology 48: 1834-1842, 2008.

11. Gallagher R: Retinoic acid resistance in acute promyelocytic leukemia. Leukemia 16: 1940-1958, 2002.

12. Sanz MA, Grimwade D, Tallman MS, et al: Management of acute promyelocytic leukemia: recommendations from an expert panel on behalf of the European LeukemiaNet. Blood 113: 1875-1891, 2009.

13. Yin T, Wu YL, Sun HP, et al: Combined effects of $\mathrm{As}_{4} \mathrm{~S}_{4}$ and imatinib on chronic myeloid leukemia cells and BCR-ABL oncoprotein. Blood 104: 4219-4225, 2004.

14. Wang L, Zhou GB, Liu P, et al: Dissection of mechanisms of Chinese medicinal formula Realgar-Indigo naturalis as an effective treatment for promyelocytic leukemia. Proc Natl Acad Sci USA 105: 4826-4831, 2008.
15. Wang N, Wang LW, Gou BD, Zhang TL and Wang K: Realgarinduced differentiation is associated with MAPK pathways in HL-60 cells. Cell Biol Int 32: 1497-1505, 2008.

16. Zhao W, Lu X, Yuan Y, et al: Effect of size and processing method on the cytotoxicity of realgar nanoparticles in cancer cell lines. Int J Nanomedicine 6: 1569-1577, 2011.

17. Janssens V and Rebollo A: The role and therapeutic potential of Ser/Thr phosphatase PP2A in apoptotic signalling networks in human cancer cells. Curr Mol Med 12: 268-287, 2012.

18. Switzer CH, Cheng RYS, Vitek TM, Christensen DJ, Wink DA and Vitek MP: Targeting SET/I2PP2A oncoprotein functions as a multi-pathway strategy for cancer therapy. Oncogene 30: 25042513,2011

19. Krajewski WA and Vassiliev OL: Histone acetylation facilitates association of nucleosomes with SET domain of ALL-1 methyltransferase in vitro. Biochem Biophys Res Commun 397: 112-116, 2010.

20. Lu J, Kovach JS, Johnson F, et al: Inhibition of serine/threonine phosphatase PP2A enhances cancer chemotherapy by blocking DNA damage induced defense mechanisms. Proc Natl Acad Sci USA 106: 11697-11702, 2009.

21. Ratcliffe MJ, Itoh K and Sokol SY: A positive role for the PP2A catalytic subunit in Wnt signal transduction. J Biol Chem 275: 35680-35683, 2000.

22. Sontag E, Nunbhakdi-Craig V, Bloom GS and Mumby MC: A novel pool of protein phosphatase $2 \mathrm{~A}$ is associated with microtubules and is regulated during the cell cycle. J Cell Biol 128: 1131-1144, 1995.

23. Kolupaeva V, Laplantine E and Basilico C: PP2A-mediated dephosphorylation of p107 plays a critical role in chondrocyte cell cycle arrest by FGF. PLoS One 3: e3447, 2008.

24. Aranda-Orgilles B, Rutschow D, Zeller R, et al: Protein phosphatase 2A (PP2A)-specific ubiquitin ligase MID1 is a sequence-dependent regulator of translation efficiency controlling 3-phosphoinositide-dependent protein kinase-1 (PDPK-1). J Biol Chem 286: 39945-39957, 2011.

25. Sablina AA and Hahn WC: SV40 small T antigen and PP2A phosphatase in cell transformation. Cancer Metastasis Rev 27: 137-146, 2008.

26. Gordon J, Hwang J, Carrier KJ, et al: Protein phosphatase 2a (PP2A) binds within the oligomerization domain of striatin and regulates the phosphorylation and activation of the mammalian Ste20-Like kinase Mst3. BMC Biochem 12: 54, 2011.

27. Guergnon J, Godet AN, Galioot A, et al: PP2A targeting by viral proteins: a widespread biological strategy from DNA/RNA tumor viruses to HIV-1. Biochim Biophys Acta 1812: 1498-1507, 2011.

28. Cristobal I, Blanco FJ and Garcia-Orti L, et al: SETBP1 overexpression is a novel leukemogenic mechanism that predicts adverse outcome in elderly patients with acute myeloid leukemia. Blood 115: 615-625, 2010 